\title{
LAS OCUPACIONES DE LOS HIJOS DE PADRES CON DISCAPACIDAD
}

Autores: Pía Alfonsina Cabello Ibarra (i), Enrique Henny Koller (ii), Ma. Carolina Hidalgo Ruedi (iii), Luis Vásquez Espinoza (iv).

\section{Resumen}

Se analizan las ocupaciones de los hijos e hijas que tienen un padre o madre con algún tipo de discapacidad y se establecen relaciones entre las ocupaciones de los hijos y la condición de su padre. El estudio se justifica por la ausencia de mayor información en la literatura de Terapia Ocupacional sobre este tema. Se utiliza un enfoque cualitativo de carácter Exploratorio, con una tendencia Explicativa. La metodología utilizada incorporó la realización de entrevistas a ocho participantes. Los contenidos de dichas entrevistas fueron transcritos y posteriormente analizados por los investigadores, quienes construyeron categorías para interpretar los hallazgos. Estas categorías fueron principalmente basadas en un marco conceptual ocupacional. Los resultados apuntan a que variadas ocupaciones son influidas por el factor discapacidad y que la realización de ocupaciones en común con los padres influye en la forma en que imaginan su futuro ocupacional en los aspectos productivos y de la crianza; en la forma en que desarrollan sus relaciones interpersonales y en la visión que tienen respecto de la discapacidad en tanto concepto y problemática social.

Palabras Claves: Discapacidad, Ocupación, Terapia Ocupacional, Hijos, Familia, Narrativa Abstract:

This paper analyses the occupations of children who have parents with disabilities and established relations between the occupations of their children and the condition of their parents. The study is justified in the absence of more detailed literature / information being available on the occupational therapy subject. The qualitative approach was characterized by an exploratory nature which provided guidance on the most effective way on how to approach the research. The methodology included, conducting interviews with eight children with parents with disabilities. The contents of these interviews were transcribed and then analyzed by the researchers who then constructed categories to interpret the findings. These categories were primarily based on a conceptual occupational framework. The results indicated that all occupations within the study were influenced by the disability factor of the parents and that this parental influencing factor also affected the way the children imagine their occupational future in the productive and parenting areas. The child(s) life perceptions are directly influenced by both the disability of the parents as well as the social interactions that a child with parents with disabilities would experience. This in turn determines how themselves develop their interpersonal relationship and the view of disability as a social construct.

Key Words: Disability, Occupation, Occupational Therapy, Child, Family, Narrative

i) Terapeuta Ocupacional, Licenciada en Ciencias de la Ocupación, Teamchess: proyecto de Canoterapia en Instituto Teletón Santiago, alfonsina127@gmail.com

ii) Terapeuta Ocupacional, Licenciado en Ciencias de la Ocupación, Académico Escuela de Terapia Ocupacional, Universidad de Chile, Fundador de Tedes Ltda. enrique.henny@gmail.com.

iii) Terapeuta Ocupacional, Licenciada en Ciencias de la Ocupación, Instituto Nacional de Rehabilitación Pedro Aguirre Cerda, caro.hidalgo.ruedi@gmail.com

iv) Terapeuta Ocupacional, Licenciado en Ciencias de la Ocupación, Comunidad Educativa y Terapéutica

"Dianova Pucara"; Centro de Rehabilitación y tratamiento CORFAPES, luis.vasquez.espinoza@gmail.com

\section{¡Error!}




\section{Introducción}

Este trabajo analiza, describe e interpreta las ocupaciones de un grupo de jóvenes que son hijos (as) de padres o madres con discapacidad, estableciendo relaciones entre las ocupaciones de los hijos y la discapacidad de sus padres y como ésta ha influido en los relatos y significados que desarrollan acerca de sus propias ocupaciones.

La investigación en la cual se basa, corresponde al Seminario de Título "Hijos de Padres con Discapacidad, descubriendo sus ocupaciones”, de Cabello, Hidalgo y Vásquez (1)

Los investigadores reconocen que entre los motivos que los llevaron a adentrarse en este tema fue la experiencia común de haber visto la película "I am Sam" (2), que relata la historia de una niña que vive con su padre con discapacidad mental y que deja entrever la forma en que esta experiencia impacta en el desarrollo de ella. Por lo que de alguna forma existía una idea previa de que la discapacidad sí impacta en la vida de los hijos, y que en muchos casos podría tener una influencia negativa, menoscabando un desarrollo típico y armónico.

Entre los motivos que justifican esta investigación se encuentran: la dificultad para encontrar información $u$ otras publicaciones acerca de este tema; la necesidad de los terapeutas ocupacionales de profundizar su mirada integral, incluyendo a la familia de las personas que se atienden, y las derivaciones que este conocimiento pudiese tener en los tratamientos diseñados y aplicados por los terapeutas ocupacionales.

Los autores perciben que no existe evidencia de que en las instituciones que brindan atención a personas con discapacidad existan programas o acciones que enfrenten la problemática de los hijos cuyos padres presentan una discapacidad. Esta ausencia de un enfoque que integre a los hijos y sus visiones de lo que ocurre con sus padres y el impacto que ésto produce en su cotidianeidad, podría estar disminuyendo la potencia de la intervención terapéutica, o dificultando la prevención de potenciales dificultades.

En este trabajo se utiliza la definición de discapacidad de la Organización Mundial de la Salud: "Toda restricción o ausencia (debido a una deficiencia) de la capacidad de realizar una actividad en la forma o dentro del margen que se considera normal para un ser humano" (3)

La población con discapacidad alcanza al $12.9 \%$, con un $51 \%$ entre los 30 y 64 años, (4) rango etáreo en el cual se conforma una familia y se vive el proceso de crianza de los hijos, lo que indica que la experiencia familiar de contar con un padre con discapacidad está presente en un número considerable de hogares, afectando la cotidianeidad personal e influyendo en la sociedad como un todo.

Uno de los supuestos de la Terapia Ocupacional es que la salud de las personas es influida por las características de las ocupaciones en las cuales participan. El conocimiento de las ocupaciones en las cuales los hijos de padres con discapacidad se involucran entregará elementos claves para la promoción de la salud en estas familias. Específicamente, las ocupaciones tendrán un impacto en la adecuación social, desarrollo de hábitos y desarrollo de la identidad (5) en los hijos, a partir de probables desequilibrios en la forma en que estas ocupaciones se experimentan. Finalmente, estas dificultades podrían constituirse en factores de riesgo en la salud de la familia y en particular de los hijos.

Entre las variadas definiciones de ocupación (Meyer (6), Willard (7), modelo canadiense (8), Clark FA(9), Nelson (10) el equipo investigador optó por basarse en la definición desarrollada por David Nelson (10) $\left(\right.$ Nelson $^{1}$ ) en la cual señala que ocupación es "una relación dinámica entre una forma ocupacional, una persona con una estructura desarrollada única, con significados subjetivos y propósitos, y el consiguiente desempeño ocupacional”

En la visión de este autor la forma ocupacional es un conjunto de aspectos físicos y socioculturales que son externos a la persona, y que se presentan en un momento del devenir de esta persona. 
La persona, quien es la que se encuentra con esta forma ocupacional, se entiende como poseedora de una estructura particular que reúne unas habilidades y características sensoriomotoras, cognitivas y psicosociales. Esta estructura se comprende en continuo desarrollo y cambio, por lo que se le llama estructura desarrollada.

Una vez producido el encuentro entre la forma ocupacional y la persona, se produce la construcción de un significado, que comprende una interpretación perceptiva, simbólica y afectiva de esa forma ocupacional. (Si bien compartimos la distinción realizada en nuestro medio por el Comité de Ciencia de la Ocupación de la Escuela de Terapia Ocupacional de la Universidad de Chile (11), en tanto distinguir significado de sentido, utilizaremos significado en un sentido amplio. De esta forma no introducimos un cambio al modelo de Nelson.) Es en este momento que esta persona puede desarrollar un propósito respecto de esa forma ocupacional, que es la experiencia de querer un resultado y por tanto de hacer algo o actuar. Si se genera un propósito, se produce el desempeño ocupacional, que es el hacer de esa persona en el contexto de la forma ocupacional.

La dinámica de este proceso tiene consecuencias tanto a nivel de la persona como de su entorno. En este modelo se entiende que los efectos de un desempeño ocupacional ocurren como resultado de un cambio en la forma ocupacional. Estos efectos ocurren tanto en la persona que ha desempeñado la ocupación, lo que se denomina adaptación, como en el medio físico y social, los que se denominan impacto.

La Terapia Ocupacional como disciplina subscribe la idea de la salud de las personas es influida por las características de las ocupaciones en las cuales participan. El conocimiento de las ocupaciones en las cuales los hijos de padres con discapacidad se involucran, puede aportar a la promoción de la salud en estas familias. En este sentido, las ocupaciones tienen un impacto en la adecuación social, desarrollo de hábitos y desarrollo de la identidad (5) en los hijos. Las dificultades en el proceso de participación en ocupaciones, podrían constituirse en factores de riesgo en la salud de la familia y en particular de los hijos.

La necesidad de prestar atención al entorno familiar, proviene de la concepción del hombre es un ser social, debido a que la mayor parte de su conducta se desarrolla en grupo y responde a estímulos de carácter social. En este proceso de respuesta, las personas adquieren las características que reflejan el contexto socio-cultural en el que se desenvuelven. En esta línea la familia es el contexto social más inmediato de cualquier sujeto, y particularmente de los hijos. La familia se constituye en un ente sociabilizador, generador de cultura y conocimientos de sus integrantes. Es evidente entonces que la familia sufre un impacto cuando uno de sus integrantes presenta una discapacidad.

Se ha definido el estrés familiar como un "estado de tensión que surge en las familias cuando el estado de equilibrio de éstas es alterado por sus transiciones a través del ciclo vital" (12). La presencia de uno de los miembros de la familia con algún tipo de discapacidad podría generar este estrés. No todas las familias reaccionarán igual, y Boss establece que esta condición está influenciada por componentes externos e internos.

Dentro de los contextos externos se considera a la cultura en la cual la familia se ha formado y vive, los ideales, las normas y valores. Por ejemplo, el contexto histórico como los períodos de dictadura, catástrofes naturales, o el contexto económico.

En el contexto interno se considera la estructura de la familia, como lo son los roles asignados y, los límites establecidos. Dentro de este contexto, el psicológico está dado por la percepción del evento traumático, la cual altera el grado de movilización de los mecanismos de defensa y de afrontamiento de la familia, que pueden ir, desde la negación hasta la aceptación de la situación estresante, y por tanto, muchas veces modifica el éxito o fracaso del proceso de solución de problemas utilizado. Por último, el contexto valórico hace referencia al sistema de creencias y 
valores utilizado por la familia para definir las situaciones conflictivas que le toca vivir, como por ejemplo, el acercamiento a la religión.

El impacto que tiene una discapacidad dentro del funcionamiento familiar altera el curso natural de las prácticas cotidianas, representando un cambio en el ciclo vital de la familia y constituyendo así, una alteración a la dinámica familiar. Este concepto de estrés familiar, probablemente no sea vivenciado directamente por los hijos, cuando la discapacidad de uno de los progenitores se ha establecido con anterioridad a su nacimiento.

En síntesis, la relevancia del presente trabajo recae en la importancia de describir e interpretar la influencia del factor discapacidad paterna, en los hijos, como producto del desempeño de determinadas formas ocupacionales, que están dadas por el contexto particular que rodea la presencia de un progenitor con discapacidad. Estos conocimientos podrían servir de base para el desarrollo de estrategias preventivas para colaborar en el desarrollo adecuado de los hijos de padres con discapacidad y sus familias.

\section{Metodología}

Se utiliza un enfoque cualitativo de carácter Exploratorio, con una tendencia Explicativa (13). En este sentido entrega "una descripción detallada de situaciones, eventos, personas, interacciones y comportamientos que son observables, incorporando lo que el participante dice, sus experiencias, actitudes, creencias, pensamientos y reflexiones, tal y como son expresadas por ellos mismos"(14). Existe una relación entre la perspectiva cualitativa y una visión sistémica en la forma de entender a las personas. Ambos ven a la persona como un todo, y no por la reducción de sus partes, dando una importancia al contexto y las experiencias de las personas. La metodología cualitativa, como lo indica su propia denominación, tiene como objetivo la descripción de las cualidades de un fenómeno. Genera un conocimiento que abarca una parte de la realidad. No se trata de probar o de medir en qué grado una cierta cualidad se encuentra en un cierto acontecimiento dado, sino de descubrir tantas cualidades como sea posible.

La elección de esta metodología pareció la más apropiada dada las complejidades del fenómeno por estudiar y la novedad de su estudio en relación al campo de la terapia ocupacional.

Se utiliza un Muestreo Intencionado y de Conveniencia, en donde los elementos a investigar y sus factores fueron seleccionados con anterioridad, y la elección de la muestra se basó en criterios prácticos, de facilidad y conveniencia para el grupo investigador (13).

La herramienta de recolección de datos fue la entrevista individual semi-estructurada, que permitió recoger el relato de vida de los participantes. Para su construcción se aplicó inicialmente una entrevista exploratoria a una de las participantes, la cual fue transcrita y analizada, de manera de utilizar la información para orientar las entrevistas subsecuentes.

Las etapas que se cumplieron fueron las siguientes:

1. Búsqueda de casos: Se contactaron instituciones que brindan atención a personas con discapacidad y se seleccionaron personas que tuviesen hijos y presentaran una discapacidad, con al menos dos años de evolución.

2. Selección de participantes: Se realizó una selección, bajo los siguientes parámetros:

a. Hijo y/o Hija de padre con discapacidad.

b. Entre los 15 y 25 años. Se consideró esta edad debido a la necesidad de contar con relatos suficientemente ricos en información.

c. Los participantes debían firmar un consentimiento informado.

3. Realización de Entrevistas: Se utilizó una grabadora de audio para registrarlas íntegramente.

4. Transcripción completa de entrevistas 
5. Análisis de las entrevistas: Se realizó una lectura y análisis inicial en forma independiente por cada uno de los investigadores que realizaron las entrevistas. Posteriormente se pusieron en común las percepciones, comentarios y factores encontrados durante su lectura y a partir de las experiencias personales de los entrevistadores durante la entrevista.

Estas conversaciones sirvieron de base para una interpretación común del grupo investigador, integrado además por un terapeuta ocupacional y un trabajador social que cumplía el doble rol de profesor guía y colaborador en el seminario de título que da origen a este trabajo.

6. Creación de cuatro categorías de análisis: A partir del proceso anterior, se definieron cuatro categorías que permitieran presentar la información generada a partir de las entrevistas.

A continuación se describen algunas características de los participantes en el estudio (ver figura 1), modificadas de manera de salvaguardar el anonimato de los participantes.

- Natalia de17 años, vive con su madre y 2 hermanos (19 y 15 años); reside en la comuna de Maipú. Su padre presentaba discapacidad de origen visual desde antes de que ella naciera. Al momento de la entrevista, Natalia no vive con su padre, ya que sus padres se separaron cuando ella tenía 15 años.

- María de 20 años, vive con su padre y madre, residen en la comuna de Nuñoa. Su padre presenta una discapacidad de origen físico por secuela de poliomielitis, que presentó durante su infancia.

- Claudio de 20 años, vive con su padre, madre y 2 hermanos (de 23 y 15 años), en la comuna de Maipú. Su padre presenta una amputación unilateral de miembro superior, desde antes del nacimiento de Claudio.

- Antonia de 22 años, vive con su padre, madre y dos hermanos de 18 y 15 años, en la comuna de Maipú. Su padre presenta una amputación bilateral de miembros superiores, desde que Antonia tenía 13 años.

- Ignacia tiene 18 años, vive con su padre, madre y dos hermanos de 22 y 15 años, en la comuna de Maipú. Su padre presenta una amputación bilateral de miembros superiores, desde que Ignacia tenía 9 años.

- Franco de 20 años, vive con su padre, madre y hermano de18 años, en la comuna de Puente Alto. Su padre presenta una amputación unilateral de miembro superior, desde antes que Franco naciera.

- Elizabeth de 24 años, vive sola, con residencia en la comuna de Las Condes. Su familia: padre, madre y hermanas de 17 y 10 años, se encuentran fuera de Chile hace 5 años. Su padre presenta secuelas de esclerosis múltiple, desde que Elizabeth tenía 16 años.

- Constanza, de 20 años, vive con su madre y hermano, reside en la comuna de Las Condes. Su madre presenta un diagnóstico de esquizofrenia paranoide, con una primera crisis cuando Constanza tenía 14 años. La última crisis se había presentado 4 años antes de la entrevista, y su mamá estaba de regreso al hogar desde hacía 7 meses. Durante el periodo crítico Constanza. vivió con su hermano y padre. Sus padres se habían separado cuando ella tenía 8 años.

Figura $\mathrm{N}^{\circ} 1$

Participantes en el Estudio

\begin{tabular}{|ccccccc|} 
Participante & Edad & Discapacidad & Madre/Padre & $\begin{array}{c}\text { Número de } \\
\text { Hermanos }\end{array}$ & $\begin{array}{c}\text { Periodo vivido junto a } \\
\text { progenitor con } \\
\text { discapacidad }\end{array}$ & $\begin{array}{c}\text { Comuna de } \\
\text { Residencia }\end{array}$ \\
Natalia & 17 & Visual & Padre & 2 & 15 años & Maipú \\
María & 20 & Física & Padre & 0 & Toda su vida & Ñuñoa \\
\hline
\end{tabular}




\begin{tabular}{|ccclccc|}
\hline Claudio & 20 & Física & Padre & 2 & Toda su vida & Maipú \\
\hline Antonia & 22 & Física & Padre & 2 & 9 años & Maipú \\
Ignacia & 18 & Física & Padre & 2 & 5 años & Maipú \\
\hline Franco & 20 & Física & Padre & 1 & Toda su vida & Puente Alto \\
Elizabeth & 24 & Física & Padre & 2 & 7 años & Las Condes \\
Constanza & 20 & Psíquica & Madre & 1 & 4 años & Las Condes \\
\hline
\end{tabular}

\section{Resultados}

Debido a las características de la metodología cualitativa utilizada, los resultados se presentan acompañados de interpretaciones explicativas, apoyadas por los relatos de los participantes.

Las categorías creadas con el fin de presentar los hallazgos fueron cuatro: Ocupaciones del cotidiano, Influencia histórica de la discapacidad de los padres en el desempeño ocupacional de los hijos y de los integrantes de la familia, el Modelamiento ocupacional y la Visión de los hijos respecto del concepto discapacidad y su impacto social.

- Ocupaciones del Cotidiano. Entendidas como aquellas ocupaciones que se desempeñan en el diario vivir en forma habitual.

En los relatos, los participantes describen que sus desempeños ocupacionales cotidianos han sido influenciados por la discapacidad del progenitor, en varias de sus ocupaciones cotidianas, tales como la alimentación, el traslado, el esparcimiento, y en las ocupaciones productivas.

Constanza, cuya madre presenta discapacidad psíquica, relata de la siguiente forma el cambio que ocurrió en el desempeño de sus ocupaciones cotidianas:

“...Es importante por ejemplo sentarnos a comer a la mesa, suena súper ridículo, es algo tan chico, pero es que antes llegaba te servías la comida enojado porque llegabas cansado veías el mugrerio en la casa cachai, yo por ejemplo con eso me colapsaba cocinaba para mi, de hecho me servía y me llevaba la comida a la pieza y comía viendo televisión y hoy día no, hoy día me gusta comer en la mesa..."

Constanza detalla en esta cita cómo su desempeño de la ocupación de alimentación presentó un cambio en los momentos de crisis de su madre. El desempeño ocupacional de las formas ocupacionales cotidianas cambia, porque la forma en sí ha cambiado, debido a que los actores expresan su propia estructura personal en ese momento y por tanto el significado construido por la persona que desempeñará potencialmente la ocupación, es influida por esta realidad.

En el desempeño ocupacional de alimentarse se observa que Constanza otorga un significado afectivo de "enojo" ante la forma ocupacional de tener que preparar su comida en un ambiente de "mugrerio". Este significado desencadena la elección de un desempeño específico de esta forma ocupacional. "... de hecho me servía y me llevaba la comida a la pieza y comía viendo televisión..." Constanza señala el cambio, producido una vez que vive con su padre, cuando señala "... hoy día no, hoy día me gusta comer en la mesa...". Conservando este desempeño ocupacional hasta su cotidianeidad actual.

El traslado, otra de las actividades cotidianas realizadas por los hijos en compañía de sus padres, produce una adaptación en la estructura desarrollada de los hijos; Natalia, cuyo padre presenta discapacidad visual, comenta:

“...De hecho el salir con él, andar en las micros, igual para nosotros era nervioso, porque nosotros igual cuando éramos chicos teníamos que salir con él, igual nos poníamos nerviosos, no sabíamos que hacer pero después nos fuimos acostumbrando y nos fue mas fácil..." 
Se puede interpretar de esta cita, que las características personales desarrolladas de Natalia, cuando enfrentó la forma ocupacional de viajar en micro con su padre, comprenden una serie de habilidades, tanto cognitivas, sensoriomotoras y psicosociales. Los desempeños que ella realizó inicialmente, le produjeron una adaptación en sus estructuras personales, de manera que ella puede percibir un cambio entre "ponerse nerviosa" y luego reconocer que con el transcurso del tiempo y la experiencia su desempeño se hacia más fácil debido a la disminución del nerviosismo y la ansiedad, que provocaba en ella el enfrentarse a la forma ocupacional del traslado en micro.

La presencia de discapacidad de uno de los padres genera cambios en las ocupaciones de ocio y tiempo libre.

Constanza señala:

“...yo le pedía permiso a mi hermana y todos queríamos carretear pa desahogarnos, era el juego del fosforito, mi hermana tenia tres fosforitos uno de ellos partido por la mitady el que sacaba el mas corto se debía quedar cuidando a la mamá, una vez me toco 3 semanas sacar el fósforo más corto, taba histérica, que era la forma de botar el estrés de hacer como que aquí no pasa na y tener que quedarme con la vieja encerrá en mi pieza jugando computador toda la noche..."

Enfrentados a la compleja forma ocupacional, que puede describirse, como salir de fiesta en el contexto de una madre con una discapacidad, Constanza se involucra en una segunda forma ocupacional relativa a la negociación entre los hermanos para decidir quien quedaría al cuidado de su madre por las noches, o en términos ocupacionales, para establecer cual sería el propósito a desarrollar, si la fiesta o el cuidado. Por tanto el desempeño de la ocupación de salir de fiesta o "carrete" puede no realizarse por la preeminencia de la forma ocupacional de cuidar a la madre. Desde otra perspectiva, en esta cita se aprecia como Constanza construye un significado ante la forma ocupacional de salir de fiesta, el que aparece como una forma de desahogo y escape de las preocupaciones que se presentaban en su hogar.

En el relato de Franco, encontramos otra forma del impacto personal del desempeño de las formas ocupacionales en el contexto de la discapacidad paterna. Al preguntarle sobre las actividades recreativas que realiza con su padre, señala:

“...Sí, su partido, después hacemos asado, también hacemos sus paseos lejos también jugamos contra otros clubes...”

Luego al indagar cual era el significado que atribuía a esta ocupación con su padre, refiere:

...Me da risa porque es pillo... con el muñón te lo empieza a poner en la boca y empieza a pegarte cortitos... pero es bueno mi papá...”

“...Bien po, contento que a pesar de su mano, igual puede jugar...”

En este caso el desempeño de esta forma ocupacional impacta en Franco en tanto el construye un significado de características positivas respecto de la presencia de la deficiencia física de su padre, que contribuye a una forma ocupacional particular.

Siguiendo con las ocupaciones del cotidiano, Elizabeth, quien tiene un padre con Esclerosis Múltiple Congénita señala:

“...en lo de los permisos me daba lata, por que por la edad en la que estás en $3^{\circ}$ y $4^{\circ}$ medio, empiezas a salir más te arreglas más por que te gusta un gallo, típico de las minas, entonces que tu papá no te deje salir o que tenía que ir con un compañero que me iba a dejar y a buscar, no podía hacer lo mismo que mis compañeros y eso me daba demasiada lata..."

En este relato la participante refiere un significado emotivo de "lata", por el hecho de que el padre no la dejase realizar las formas ocupacionales de la manera en que a ella le hubiese gustado. La influencia de la discapacidad en la forma ocupacional se hace presente en el siguiente párrafo: 
“...heee era complicado, mi papá se puso muy sobre protector, incluso me dejaba salir con un solo compañero que era el que me tenía que ir a buscar y a dejar, y a tal hora, y se empezó a preocupar mucho más y se puso más sensible, por que le venían los dolores y tu no sabías y el andaba irritable y se peleaba con la gente que no debía ser..."

De esta manera, podemos interpretar que la forma del desempeño ocupacional del padre ante las ocupaciones relacionadas con el cuidador de sus hijos, se afectaron por su condición, lo que a su vez impacta en las formas ocupacionales presentadas a Elizabeth y en su estructura personal:

“... sip, de todas maneras, como que me atrasé en todo. Ponte mi primer beso fue a los 18 años, mi primer pololo duro como 3 años, me atrasé en todo lo que iban mis compañeras, por permisos me volví como más introvertida...”

La condición de discapacidad del padre también impacta en las formas ocupacionales relacionadas con la ayuda del hijo al padre. En las que el desempeño, el propósito y el significado resultan afectados. Esto se visualiza en el relato de Claudio:

“...No, pocas, a cortar la carne, destapar algún frasco, le ayudo a cortar la carne, pero muchas veces me pide ayuda de flojo, yo me doy cuenta, uno conoce a los papás.

Entrevistador: ¿Cómo te das cuenta?

Por las caras que pone, o porque muchas veces eso mismo que pide ayuda, lo ha hecho solo antes. Además si le preparan carne, ésta es finita para que la corte él solo..."

Claudio declara que el ayuda a su padre, participando en ocupaciones comunes. Sin embargo, él aprovecha la oportunidad en la entrevista para expresar que su padre es capaz de hacer la mayoría de las cosas, y por tanto cuestionando su cualidad de persona con discapacidad. Por otra parte, se podría interpretar que Claudio asume un papel activo para favorecer las capacidades del padre, dándose cuenta de las veces que él pide ayuda, sin realmente necesitarlo.

En síntesis, la discapacidad del padre influencia la estructura desarrollada de los hijos, a través de la modificación de las formas ocupacionales, los significados construidos y los propósitos, expresados en los desempeños ocupacionales cotidianos vividos en el contexto de la discapacidad de sus progenitores.

- Influencia histórica de la discapacidad de los padres en el desempeño ocupacional de los hijos y de los integrantes de la familia. Categoría que se utiliza para describir el antes y el después de la presencia de la discapacidad, en la vida de los participantes.

Las formas de desempeño y las formas ocupaciones que se han identificado en la mayoría de los participantes presentan un antes y un después de la discapacidad del progenitor. Los hijos que tuvieron desde siempre a un padre con discapacidad, presentan una estructurada desarrollada diferente a aquellos cuyos padres adquirieron la discapacidad posteriormente.

María, hija de un padre con discapacidad física expresa:

“...cuando viajamos, nos prepararnos nosotros y preparamos al resto... tenemos que decir vamos a ir con una persona lisiada, por ejemplo con los aviones el primer asiento es más grande que los de atrás, con mi mamá peleamos por ese asiento, por 2 asientos, pero tenemos que luchar y tener cuidados por ejemplo al pedir un hotel, debe tener ascensor o si no en un primer piso..."

María ve estas formas ocupacionales como normales en su vida. La ocupación es ir de viaje, las características del hotel como el poseer ascensor y habitaciones en el primer piso, constituyen la forma ocupacional de esta ocupación, como lo es también el viajar en un avión donde se requieren asientos amplios. Si bien estas son de alguna manera adaptaciones que son necesarias para ese padre con discapacidad, su visión o significado, es de normalidad, porque así 
lo ha vivido siempre. Por tanto el ir de vacaciones en familia, aunque diferente en su forma, persiste sin experimentar un cambio importante.

La vivencia y experiencia de esta forma ocupacional y otras más, han influido en la desarrollo de su estructura personal, principalmente en sus aspectos cognitivos y psicosociales, generando una conciencia particular hacia las personas con discapacidad, otorgándole un significado diferente a otras personas que no tienen la experiencia o vivencia con alguna persona con discapacidad, reflejándose en otro de sus relatos:

“...en cuanto a lo de los estacionamientos o el supermercados distinto es, por que a mí también me daba rabia, muchas veces iba yo manejando sin mi papá y le decía al guardia que no podía ser, o veía alguien que se estaba estacionando y le decía yo no veo que tenga ningún problema y le alegaba, de hecho me agarré varias veces con señoras por lo mismo...

también me he dado cuenta que en Chile la cultura con las personas con discapacidad no se respeta tanto, por ejemplo los estacionamientos para lisiados siempre están llenos, por ejemplo, o cosas así..."

María menciona explícitamente que su vivencia con la discapacidad de su padre ha sido más bien algo que la ha "acompañado en la vida":

“..la discapacidad de mi papá es como, bueno para mí desde que nací está, o sea, no es como algo que marcó mi vida, como una cosa que siempre me ha acompañado en la vida y para mí no me complica no sé po ayudar a una persona que esta en silla de ruedas a cruzar la calle, para mi no es como algo nuevo por que si tengo que hacerlo lo hago, bueno me acuerdo cuando voy a misa y hay abuelitas que andan en silla de ruedas, me preocupo de correr las sillas para mi es demasiado normal eso, como preocuparme, como que eso gente de afuera me lo dice, pero siempre cuando voy a misa o cosas así, me preocupo de donde se va a poner que no hayan sillas, como de correrle las sillas, por que estoy acostumbrada ha hacerlo con mi papá ..."

Por otra parte, se encuentran los hijos que vivieron con sus padres antes que tuvieran una discapacidad. En este caso, el tipo de discapacidad y características específicas de la misma parecen tener una gran importancia.

Constanza menciona como sus ocupaciones se ven estrechamente relacionadas con los diferentes ciclos que cursa la enfermedad de su madre, lo que se observa en el siguiente relato:

“...todos los años a Algarrobo arrendábamos la mismas casa todo, todo igual todos los años, hasta que mi mamá se enfermó, de hecho mi papá pagaba las vacaciones, él se fue y seguía pagando las vacaciones independiente, pero mi mamá se enfermo y no tenía sentido irse a Algarrobo, porque mi mamá hacia todo, en el fondo no tenía vacaciones, mi mamá iba y se dedicaba a atendernos, entonces después se enfermó y no tenía sentido... igual éramos chicos, mi hermana manejaba, pero no era lo mismo..."

Se observa que la forma ocupacional de ir de vacaciones y por ende el desempeño ocupacional de la hija y su familia tuvo un cambio importante y fue influenciada por un antes y un después de la discapacidad de la madre.

Otras formas ocupacionales cotidianas, influenciadas por el antes y después de la discapacidad, es la que se encuentra en los siguientes relatos de Constanza, cuya madre tiene una discapacidad de origen psíquico:

“...El computador en la otra casa tenía su pieza, estaba la pieza del computador, ahora ponemos el computador en el living para jugar con mi hermano, conectamos los computadores y jugamos juntos y antes nadie estaba en el living, habiendo tele en el living, habiendo de todo, nadie quería estar en el living, todos querían estar encerrados..”.

“... me daba lata ir, me daba lata llevar a mi mamá al dentista, mi papá me pasaba cien lucas, entonces lo que yo hacia era ir a buscarla a la clínica tomaba un taxi, llegábamos al dentista, la esperaba afuera y después tomábamos un taxi y la dejaba en la clínica y me iba para mi casa...cuando mi mamá estuvo mejor, me iba en micro, me llevaba a mi mamá en micro al 
dentista, nos íbamos conversando, llegábamos antes pasábamos a tomarnos un café o un helado comprábamos algo para comer, nos íbamos al dentista, yo entraba con ella a la consulta, veía todo lo que le hacían, después nos íbamos a comer algo rico ... Pero cuando era una obligación, era horrible para mi era una lata, no quería hablar con ella. Cuando recién le pasó, ella se estaba armando un rompecabeza, era hablar de la enfermedad, era escuchar todavía a una persona enferma, era encontrar a la persona que tenia perdida hace 4 años. Siempre he dicho que mi mamá enferma es mi mamá física, pero no es mi mamá, es un cuerpo poseído por otro ente que yo no conozco que me odia que yo odio y que es horrible y mi mamá sana es mi mamá, con la que yo puedo conversar, que la abrazo, que la quiero, la adoro y todo, entonces había harta diferencia entre cuando ella estaba bien y cuando estaba mal...”

Este relato refleja por una parte el cambio que ocurre en la forma ocupacional del traslado, en taxi o en metro, influido por la situación de discapacidad. Pero por otra parte, y de manera dramática, refleja la diferencia en el significado afectivo que Constanza experimenta dependiendo del momento de la enfermedad de su madre. En un caso es un sentimiento de agrado y en el otro de rechazo y obligación al momento de ejecutar el papel de cuidador de su madre.

Este es el único relato obtenido en esta muestra que coincide con una de las ideas previas del grupo investigador, el cual se refería a que la discapacidad produciría un impacto negativo en los hijos, por el hecho de la adopción de un papel de cuidador de los padres.

El presentar socialmente a su padre a otras personas es una actividad cotidiana a la que se ven expuestos los hijos. Esto comprende explicar a otros aspectos de su vida familiar, el tiempo que su padre presenta una discapacidad o los motivos de la misma. De esta forma, una actividad cotidiana común y sencilla para la mayoría de las personas se constituye en una oportunidad para reflexionar sobre el significado valórico de vivir con un padre con discapacidad, ya que las respuestas que incorpore en su discurso constituirán una nueva experiencia interpretativa que incluirá sentidos personales de vergüenza, alegría y/o orgullo, lo que se ilustra con los siguientes relatos.

Elizabeth señala lo siguiente:

“...en lo de los permisos me daba lata, por que por la edad en la que estas en $3^{o}$ y $4^{o}$ medio, empiezas a salir más te arreglas mas por que te gusta un gallo, típico de las minas, entonces que tu papá no te deje salir o que tenia que ir con un compañero que me iba a dejar y a buscar, no podía hacer lo mismo que mis compañeros y eso me daba demasiada lata..."

María señala en estas tres frases, el valor significativo y el sentimiento de orgullo al presentar a su padre a los demás, en los siguientes relatos:

“...nunca fue como...ayy que lata que mi papá sea así, nunca me lo plantié, a ver yo siempre lo viví así... creo que alguna vez le he preguntado a mi mamá ¿qué pasó?, el por qué mi papá era así, pero no se cual pudo haber sido esa respuesta. Pero nunca fue como ohh, que lata que mi papá sea así..."

“...obviamente uno llama más la atención, obvio...”"

“... Ahora también lo veo como que es mi realidad y pucha bacán cachai, y pucha todo el mundo que me conoce, lo más cercanos saben que mi papá es el referente de mi vida...”

Natalia, por otra parte señala que:

“... como que a todos les da pena, pucha tu papá no ve, tu papá es cieguito. Me molestaba que tuvieran pena de él, pero también había gente que hasta el día de hoy admira a mi papá por todos los logros que ha tenido siendo que es discapacitado. Yo encuentro que mi papá no es una persona que merezca lástima o cosas así po..."

No obstante, existen otros dos relatos sobre la estructura desarrollada de los hijos al momento de presentar a su padre con discapacidad. Este es el relato de Antonia y Constanza respectivamente, la primera cuyo padre tiene una discapacidad física y la segunda cuya madre tiene una discapacidad psíquica. 
“...No, nunca me ha dado vergüenza mi papá, yo siempre se los presento a todos mis conocidos, y les cuento altiro lo que le pasó, así que todos saben y nadie nos pregunta nada.”

“... hubo un tiempo que yo no quería llevar gente a la casa, mi pololo cuando fue dos o tres veces a mi casa, eso que llevábamos 2 años pololeando, una vez entró porque me tenía que ayudar a mover un mueble y la otra porque había un asado.... yo tenía vergüenza de mi mamá, esa es la palabra, de manera súper fría, eso era, como que me daba pena que me tuvieran pena, que dijeran pucha pobrecita la vida que le tocó llevar. Por eso me daba vergüenza que conocieran a mi mamá, que ella hiciera el ridículo y en el fondo... a todos le importa lo que digan los demás..."

Existen por otro lado, ocupaciones que se dejan de desempeñar por una mala experiencia relacionada con la discapacidad de su madre, en este caso en particular, como lo es el "carretear" (irse de fiesta). Constanza refiere ante esto lo siguiente:

“... hubo un tiempo que fumaba pitos, jalaba de repelente y tomaba mucho y corte esto porque el psiquiatra una vez me dijo que por un pito quizás hoy día no me pasaba nada, pero el día de mañana sí... porque hay un gen en mi que esta esperando saltar en el fondo y como esperando desatar, como que me podía pasar, entonces yo corté todo eso, igual tomo, pero ya no fumo marihuana ni por si acaso. Desde 4to medio que no fumo y na po. Lo otro que hacemos es que mi mamá se le detonó todo por unas pastillas para adelgazar entonces, yo con suerte tomo paracetamol..."

“... he dejado de hacer las cosas negativas como carretear, yo creo que lo hacia para resguardarme y hacer como aquí no pasa nada de demostrar que era una chiquilla feliz, ahora no tengo la necesidad de salir a carretear, ahora voy a la casa de mi pololo a ver películas, además se queda en mi casa, sí...jva a mi casa!, ya no es un ambiente tétrico esta casa nueva tiene un ambiente súper familiar...”

Se observa que Constanza ha sido la única hija, dentro de esta muestra, que ha optado por no proponerse ciertas ocupaciones por la discapacidad de su madre. Otros relatos van en otra dirección. Elizabeth refiere lo siguiente:

“... no me he limitado, porque creo que nosotros lo motivamos a él, a veces le exigimos que lo haga por que sabemos que lo puede hacer, pero no, nunca yo me he limitado..."

En síntesis, los aspectos ocupacionales que más cambian con la discapacidad de lo progenitores y el contexto socio histórico, son los significados que le otorga cada hijo a determinadas ocupaciones, lo que está influenciado por su propia estructura desarrollada, que es única y subjetiva, construida por sus experiencias junto a sus padres.

- Modelamiento del presente y futuro ocupacional de los hijos como consecuencia de la discapacidad de los padres

Las relaciones y acontecimientos que existen dentro de la familia, influirían en la formación de los hijos y en su futuro. La mayoría de las ocupaciones que realizan en la actualidad los participantes tienen un grado de influencia por la discapacidad del padre. Asimismo, esta experiencia modela el como imaginan y ven su futuro ocupacional. Como lo refiere Nelson, el "aprendizaje comprende el proceso de adaptación y siempre es el resultado de una ocupación" (10). Algunos hijos, señalan que la elección de su carrera, está directamente influenciada por la discapacidad del padre.

Antonia, hija de un padre con discapacidad física, señala lo siguiente:

“... Tengo la influencia de mi padre por los trabajos de hombre jejeje. Él me enseñó arreglar artefactos eléctricos, como lo son los enchufes, lámparas, etc... Por eso es que estudié aeronave y trabaje 2 años. Luego lo dejé cuando fui madre, pero quiero seguir trabajando en trabajos de hombre, en trabajos de maestro de chasquilla, que es lo que me gusta...”

Por otra parte Constanza, hija de una madre con discapacidad psíquica, señala: 
“Entré a estudiar trabajo social para ayudarla... yo creía que iba a poder eso sí, pero de ahí, me di cuenta que no, me di cuenta que era imposible, no se puede intervenir a un familiar, no tiene sentido".

En ambos relatos, la elección de un estudio futuro está influenciada por la discapacidad del padre. Primeramente, Antonia refiere la necesidad de ayudar a su padre en los trabajos que él no podía realizar. Esas experiencias producen una adaptación personal que cambia su estructura, lo que parece afectar directamente la elección de su carrera profesional. Constanza, con la elección de su carrera, tuvo en una primera instancia el deseo de ayudar a su madre. Al avanzar los años de su carrera, Trabajo Social, se dio cuenta en qué consistía y que no iba a poder ayudar a su madre como ella deseaba, pero esta conciente que podrá ayudar a otras personas que presentan situaciones parecidas a las de su madre.

María, hija de un padre con discapacidad física, agrega lo siguiente:

“.. Por que siempre he tenido el sueño. ahh porque hago obras sociales siempre, entonces mi idea es estudiar una carrera y crear una fundación, pero para crear una fundación tienes que ser ingeniero, ingeniero comercial, conseguirte plata, etc. ponte no era para ponerme ha estudiar trabajo social, mucha gente me decía ahh, pero ponte a estudiar trabajo social, el nombre dice que la carrera es muy social, pero no tiene nada que ver con lo que quiero hacer. Por eso quería algo con las matemáticas que también te sirve para empresas y que sirve para empresa, ingeniería no más, así como para crear cosas grandes...”.

En ella, los motivos para elegir su carrera se relacionan con la aspiración de formar una fundación para ayudar a las personas con algún tipo de discapacidad. En términos ocupacionales, la experiencia de vida con su padre con discapacidad, introdujo significados particulares y propios a la ocupación de elegir una carrera. A su vez, se aprecia que la forma en como se enfrenta a la sociedad es de manera mayormente critica, relacionado esto con una sensibilidad ante este tema.

En el transcurso de la investigación, los participantes refieren una fuerte admiración por su imagen paterna, lo que hace plantearse en ellos el cómo desempeñaran sus ocupaciones cuando sean padres.

Refiriéndose al tema de la futura crianza de los hijos, Antonia, hija de un padre con discapacidad física, menciona:

“... El hecho de tener un hijo, lo ha puesto más feliz a mi papá y esta chocho con él. Yo ahora sé y admiro la fuerza que tuvo mi papá para con nosotros, la valentía que tuvo para seguir con nosotros y formar su familia. Con mi hijo puedo darme cuenta que él sacaba fuerzas de no sé donde, porque es complicado ser padre y más si uno tiene algún problema o accidente, pero él siempre se las ingenia para hacer las cosas a de lugar...".

Por otra parte Elizabeth, hija de un padre con discapacidad física, señala:

“...No voy a ser tan estricta, por que en vez de quedar como una bonita experiencia quede con un trauma, entonces trataría de hacerla como por otro lado, lo mismo que mi papá me enseño cuando chica, por que me enseño mucho, lo haría con mis hijos por que me daría lata que fueran inútiles o mantenidos. Por ejemplo que sepan si están los dos cablecitos en el techo, que sepan conectarlos o una lámpara, por ejemplo. Me ha tocado que mis compañeros no saben hacer las bolsas de ensalada y llaman a la mamá... a mi me daría vergüenza..."

Las ocupaciones realizadas en conjunto a su padre, si bien fueron impuestas por su padre y no fueron de total agrado para ella al extremo de señalar que le crearon un "trauma", dieron lugar a la posibilidad de revertir este sentimiento a uno de agrado.

Los relatos antes mencionados aluden a la forma en la cual los participantes visualizan su futuro ocupacional de ser padres y reafirman el querer realizar ocupaciones en conjunto a sus hijos.

Este es otro ejemplo de como realizaban ocupaciones en conjunto a sus padres, las que despertaban emociones positivas o negativas y que pueden cambiar de una a otra con el tiempo. 
El modelamiento ocupacional construido por los participantes no solo se ve explicitado en la ejecución o imaginario de sus ocupaciones, sino que además tienen una fuerte influencia en las relaciones interpersonales influenciadas por las experiencias vividas con una persona con discapacidad.

- Visión de los hijos respecto del concepto discapacidad y su impacto social.

Discapacidad es un término utilizado ampliamente por los terapeutas ocupacionales y por la sociedad. En el transcurso de esta investigación ha sido posible descubrir el significado que los participantes atribuyen al término, y en particular a la forma en que este término se refiere a sus padres.

En el proceso de análisis, se descubrió que los participantes mencionaban que sus padres no presentaban discapacidad, sino una enfermedad, y que en su percepción les parecían normales, en contraposición a discapacitados.

María señala:

“...a mí la palabra discapacidad no me gusta, por que la palabra discapacidad significa no capaz, la encuentro fea, por que un discapacitado no es que no sea capaz, tiene una falencia, o no se como se dice, pero tiene algo que yo no tengo, ósea tiene algo menos, cachay, pero encuentro que la palabra discapacidad que la usa todo el mundo es fea, pero filo todo el mundo la ocupa..."

Constanza señala:

“...Yo creo que es una palabra muy mal usada porque son capacidades diferenciadas. No es un discapacitado porque es decir incapaz. Porque es una persona con capacidades diferenciadas, pero eso... discapacidad, dificultad de vida eso es discriminación heavy y desprotección. Nada positivo, nada positivo...”

La percepción que tienen estas personas sobre el término discapacidad hace referencia al no poder realizar algo, estando muy ligada a su percepción de la funcionalidad de la persona. A su vez, perciben un mal uso del término, dejando entrever que no es un término de su agrado, pero que es una calificación otorgada por la sociedad a sus propios padres.

La mayor parte de los participantes relacionan la palabra discapacidad con una condición física, como un estigma, y prefieren usar la expresión "mi papá tiene una enfermedad".

Constanza relata al preguntar si es que ella asocia la palabra discapacidad con su madre, responde lo siguiente:

“...No, sabes que me acuerdo de un compañero que era discapacitado o con capacidades diferenciadas que no podía mover de la cintura para abajo, entonces estaba en silla de ruedas. Si me dices discapacidad me acuerdo de él, pero con mi mamá no, y esa es la persona más cercana discapacitada que he tenido en términos físicos no lo asocio con discapacidad mental. Me hace sentido con discapacidad física..."

Al indagar sobre la percepción positiva de sus padres, que de alguna manera expresa las capacidades, y no sus discapacidades, refieren lo siguiente:

Natalia:

“...no se si es porque es mi papá pero yo lo veo que tiene tanta fuerza que sabe tan bien salir adelante, que me gustaría tener esa capacidad que tiene él, porque él teniendo un problema no se encierra en él sale de él, porque siempre yo había pensado a mí me hubiera pasado de quedar no vidente a mi no me hubieran dado mas ganas de vivir, o sea, yo no sabría como vivir siendo no vidente, y él se maneja terrible bien yo lo admiro a él, igual nosotros a veces nos vendamos los ojos para andar en la casa y chocábamos con cosas y nos tropezábamos para ver y sentir el mundo de mi papá...”

En este relato, Natalia relata la admiración que siente por su padre y menciona que ella en conjunto con sus hermanos participaba en una ocupación que consistía en vendarse los ojos, como 
una forma de ponerse en el lugar del padre. En este caso, una forma ocupacional particular ha sido creada para sentir lo que percibe su padre. Es claro que estas ocupaciones producen una adaptación en las estructuras desarrolladas de los hijos.

Constanza relata:

“...Yo creo que de mi mamá me gusta su capacidad de amar, eso es lo más lindo de mi mamá, es tan buena que llega a parecer tonta de lo buena que es, mi mamá es buena de verdad, obviamente igual tiene sus problemas con algunas personas, pero no todo el mundo le cae bien, pero mi mamá eso tiene que es súper buena para perdonar es eso una de las mayores virtudes de mi mamá yo creo. Mi mamá es como buena de esencia, no es una persona que ande con las weas por la espalda y ande pelando por la vida, mi mamá es buena...

Los participantes demuestran una mayor conciencia ante la problemática social sobre la discapacidad, lo que atribuyen al hecho de haber vivido experiencias con sus padres y de ver su lucha por sus derechos. Desde una perspectiva ocupacional es la estructura desarrollada la que ha cambiado.

Antonia refiere:

“...Uno ve en la calle gente que pide y le da rabia, yo digo como mi papá tiene lo que tiene si tener que llegar a esas condiciones...”

\section{Conclusiones}

Este trabajo ha descrito e interpretado las experiencias vitales de un grupo de personas, que vivieron con un progenitor que portaba una condición discapacitante. El uso del Marco Conceptual de la Ocupación Terapéutica de David L. Nelson resultó práctico y esclarecedor permitiendo utilizar un lenguaje propio de la disciplina en el estudio de una experiencia de vida. Es claro que un uso de este marco por otros investigadores, permitirá continuar avanzando en la construcción de conocimiento respecto de este caso particular, así como acerca de la necesidad de contar con un lenguaje que permita expresar la visión única respecto de la ocupación.

Algunos factores, relacionados tanto con las formas ocupacionales, en particular aquellos contextuales, como con las estructuras personales de los participantes y sus familias influyen concomitantemente con el factor de la discapacidad del padre. Estos factores son los siguientes: ciclo vital, género del progenitor, carácter del padre y de los hijos, tiempo disponible para encontrarse, modernidad y separación de los padres, todos los cuales podrían ser investigados a futuro en relación a esta población.

Este grupo investigador considera que la influencia de la condición de los progenitores en cuanto discapacidad se expresa desde una perspectiva ocupacional en las cuatro categorías utilizadas para describir los hallazgos, esto es en las ocupaciones del cotidiano, en el transcurso vital de los participantes, en la forma en que perciben y hacen opciones en relación a la forma de desempeñar ocupaciones y planear su futuro ocupacional y finalmente en sus concepciones respecto de la discapacidad, en sus vertientes conceptuales y sociales.

Una de las lecciones aprendidas durante esta investigación, es el hecho de que, por lo menos en este grupo de personas, si bien experimentaron periodos difíciles y estresantes, se produjeron efectos que podrían mencionarse como beneficiosos para ellos mismos y para el grupo social al cual pertenecen. En particular, la idea preconcebida de los autores en relación a que la condición de sus padres los llevaría a tomar el papel de cuidadores y con ello menoscabar su desarrollo personal, fue desmentida por los relatos.

Parece conveniente que los terapeutas ocupacionales consideren los hallazgos de esta investigación con el fin de expandir la comprensión de las ocupaciones en situaciones de discapacidad, no sólo en relación a las personas directamente implicadas con ella, sino también en las de su entorno más cercano y en este caso los hijos. Los elementos identificados en esta investigación podría servir de base para la búsqueda y desarrollo de estrategias de intervención, 
que desde ya apuntan a una construcción individual o personalizada en cada uno de los casos y a aspectos preventivos y potenciadores de la acción terapéutica.

\section{Bibliografía}

1.- $\quad$ CABELlO, P. HIDALGO, MaC. VASQUEZ, L. "Hijos de Padre con Discapacidad; Descubriendo sus Ocupaciones", Seminario de Titulo para optar al grado académico de Terapeuta Ocupacional, Universidad Mayor, 2007.

2.- $\quad$ I am Sam (Yo soy Sam, Mi nombre es Sam o Lección de amor), es una película estadounidense del año 2001. Protagonizada por Sean Penn y Michelle Pfeiffer y escrita por Kristine Johnson y Jessie Nelson. tiraMillas.net (03 de Marzo de 2002). Recuperado el 15 de Diciembre de 2007, de Yo soy Sam: http://www.tiramillas.net/cine/2002/yosoysam/sam.html

3.- $\quad$ EGEA G, C., \& SARABIA S, A. Clasificaciones de la OMS sobre discapacidad. Murcia, 2001.

4.- $\quad$ INSTITUTO NACIONAL DE ESTADISTICA, INE. Primer Estudio Nacional de la Discapacidad en Chile. ENDISC-CIF. Chile, 2004.

5.- $\quad$ CHRISTIANSEN CH.The 1999 Eleanor Clarke Slagle Lecture. Defining lives: occupation as identity: an essay on competence, coherence, and the creation of meaning. Am J Occup Ther. 1999 Nov-Dec;53(6):547-58.

6.- $\quad$ MOSCOVICI, S. Psicología Social. Barcelona: Paidós, 1999.

7.- $\quad$ WILLARD, H. S. Terapia Ocupacional. Médica Panamericana, 2001.

8.- $\quad$ DE LAS HERAS, C., CASTRO, D., \& MIRANDA, O. Modelos de intervención basados en la ocupación; modulo de trabajo para la asignatura. Santiago de Chile, 2006.

9.- $\quad$ CLARK FA, PARHAM D, CARLSON ME, FRANK G, JACKSON J, PIERCE D, WOLFE RJ, ZEMKE R. Occupational science: academic innovation in the service of occupational therapy's future. Am J Occup Ther. 1991 Apr;45(4):300-10.

10.- NELSON, D. L., \& THOMAS, J. J. Occupational Form, Occupational Performance, and a Conceptual Framework for Therapeutic Occupation, 2003.

11.- COMITÉ DE CIENCIA DE LA OCUPACIÓN DE LA ESCUELA DE TERAPIA OCUPACIONAL DE LA UNIVERSIDAD DE CHILE. Revista Chilena de Terapia Ocupacional, p 79, Número 7, Noviembre 2007.

12.- $\quad$ FICHTER, J. H. Sociología. Barcelona: Herder, 2001.

13.- $\quad$ TAYLOR, S.J, BOGDAN, R., "Introducción a los Métodos Cualitativos de Investigación", Editorial Piados, 1987.

14..- PÉREZ S, GLORIA: "Investigación Cualitativa. Conceptos e Interrogantes”; Editorial La Muralla s.a, 1994

\section{Agradecimientos}

A los participantes que contribuyeron con sus testimonios en el desarrollo del Seminario que da base a este artículo.

A la Escuela de Terapia Ocupacional de la Universidad Mayor. 